\title{
PERFIL CLÍNICO DE NEONATOS INTERNADOS EM UMA UTI DO SUL CATARINENSE
}

\section{Clinical profile of newborns admitted on a south catarinense ICU}

Josiani Mondardo Sávio르. Cecília Marly Spiazzi dos Santos², Rozilda Lopes de Souza ${ }^{2}$, Cristiane Damiani Tomasi ${ }^{2,3}$

${ }^{1}$ Acadêmica do Curso de Enfermagem, Universidade do Extremo Sul Catarinense UNESC. Criciúma - SC.

${ }^{2}$ Curso de Enfermagem Universidade do Extremo Sul Catarinense - UNESC. Criciúma - SC.

${ }^{3}$ Núcleo de Estudos e Pesquisas em Integralidade e Saúde - NEPIS, Universidade do Extremo Sul Catarinense - UNESC. Criciúma - SC.

\section{Endereço para correspondência:}

Cristiane Damiani Tomasi

Av. Universitária, 1105 - Bairro Universitário CEP: 88806-000 - Criciúma-SC.

Email: cdtomasi@unesc.net 


\title{
Resumo
}

Estudo com objetivo de identificar o perfil clínico de neonatos internados em uma Unidade de Terapia Intensiva Neonatal (UTI-N). Pesquisa de abordagem quantitativa e descritiva. O estudo foi desenvolvido em um Hospital de Médio Porte da Região Sul de Santa Catarina. A amostra estudada foi através de documentos arquivados no local do estudo, na Unidade de Terapia Intensiva Neonatal. A análise dos dados foi realizada a partir da base de dados, através da ordenação, classificação e análise final dos dados pesquisados. Para tanto, foram coletados através de questionários contidos em 18 prontuários o correspondente no período de Outubro a Dezembro de 2014 de recém-nascidos que estiveram internados na UTIN. As análises foram feitas através de estatísticas descritivas, com índice de significância $p<0,05$. Houve associação estatisticamente significativa entre uso de fototerapia e menor idade gestacional $(p=0,017)$. As principais causas de internação na UTIN foram insuficiência respiratória, sepse e cardiovascular, as mães realizaram em torno de 6 consultas prénatal. Neste estudo é possível concluir que a menor idade gestacional está associada a necessidade de fototerapia em recém-nascidos.

Palavras-chave: Neonatos; UTI Neonatal; Fototerapia; Idade Gestacional.

\begin{abstract}
The aim of this study was to identify the Clinical Profile Newborns Hospitalized in a Neonatal Intensive Care Unit (NICU). Study with descriptive design and quantitative approach. The study was conducted in a Midsize Hospital in the Southern of Santa Catarina. The sample was through documents at the study site, the Neonatal Intensive Care Unit, the analysis was performed from the database through ordering, classification and final analysis of the surveyed data. Data were collected through questionnaires contained 18 records in the corresponding period of October to December 2014 newborns who were admitted to the NICU. To descriptive statistics where adopted a significance level $p<0.05$. The association between phototherapy and gestational age was statically significant $(p=0,017)$. The main causes of admission on NICU was respiratory failure, sepsis and cardiovascular. The mothers did around 6 prenatal visits. This study concludes that lowest gestational age is associated with newborn phototherapy need.
\end{abstract}


Keywords: Newborns; NICU; Phototherapy; Gestational Age.

\section{INTRODUÇÃO}

Os óbitos neonatais são reconhecidos universalmente como os mais importantes desfechos, sendo o baixo peso ao nascer e a prematuridade causas importantes desses óbitos. Mesmo com evidências de redução na mortalidade neonatal, os índices continuam altos, em comparação aos países desenvolvidos. Deve-se ter atenção direcionada para o controle do nascimento do recém-nascido prematuro e do recém-nascido de baixo peso ao nascer, garantir adequada acessibilidade e utilização mais efetiva do conhecimento científico e tecnológico, oferecendo assim um atendimento mais qualificado. É importante salientar que de nada adianta um atendimento de alta complexidade se não é dada a atenção necessária à gestante no período pré-natal, período perinatal, bem como no atendimento ao recém-nascido na sala de parto'.

A mortalidade neonatal pode ser determinada por diversos fatores, mas muitas causas são consideradas evitáveis. Com o conhecimento e a tecnologia existentes, é possível intervir de maneira eficaz, evitando o óbito. Mortes por causas evitáveis podem ser importante instrumento de monitorização e avaliação dos serviços de saúde. As mortes evitáveis relacionadas ao recém-nascido são reduzíveis pela adequada atenção à mulher na gestação, ao parto e ao recém-nascido, o que ressalta a importância da assistência à saúde prestada durante a gestação².

Apesar dos avanços na assistência à gestante e ao recém-nascido, as internações em unidade de terapia intensiva (UTI) neonatal constituem uma grande preocupação à equipe de saúde e aos familiares do recém-nascido, sendo as características da gestação e do parto fatores determinantes das condições de evolução do recém-nascido. A assistência prestada em UTI neonatal também vem experimentando grande desenvolvimento, porém a distribuição dos leitos não segue uma equidade. Para tanto consideram-se as desigualdades regionais do país, que acaba ofertando mais leitos em grandes centros, pela complexidade do serviço e seu alto custo de manutenção, tendo uma estrutura desigual e consequentemente acesso limitado da população ao serviço ${ }^{3}$. 
Desta forma é importante conhecer o perfil clínico dos neonatos internados em UTI, condições referentes às mães de neonatos que necessitam de leitos em UTIN, para poder intervir mais precocemente em fatores que tenham relação com esta condição, promovendo a saúde da mulher e da criança. Este estudo teve como objetivo identificar o perfil clínico de neonatos internados em uma UTI do sul catarinense.

\section{METODOLOGIA}

Trata-se de uma pesquisa de abordagem quantitativa, do tipo descritiva, documental, retrospectiva, realizada em uma UTI Neonatal de um hospital da região sul de Santa Catarina. O hospital possui 10 leitos de terapia intensiva, sendo 7 leitos para recém-nascidos (RNs) em período neonatal e 3 leitos pediátricos. Este serviço é referência de atendimento dos municípios da região da AMREC (Associação dos Municípios da Região Carbonífera) oferecendo suporte também para outros municípios de Santa Catarina.

Para o estudo foram incluídos todos os registros de neonatos internados em UTI no período de outubro a dezembro de 2014. Através de questionário padronizado foram coletados dados sobre a gestação e o parto, bem como dados clínicos da internação do RN. O estudo foi aprovado pelo Comitê de Ética em Pesquisa do local de origem do estudo (parecer número 999.996/2015).

Após a coleta de dados foi realizada a inserção dos dados em planilha eletrônica, para posterior análise estatística. As comparações de variáveis categóricas foram realizadas pelos testes Exato de Fisher e Qui-quadrado (com a correção de Yates quando indicado), além da apresentação por meio de frequência absoluta e relativa. As variáveis contínuas foram apresentadas na forma de média \pm desvio padrão e comparadas com o teste t-Student. Em todas as análises, foi adotado como nível para significância estatística um valor de $p<0,05$. Os testes estatísticos foram realizados pelo software estatístico SPSS 22.0 IBM®. 


\section{RESULTADOS}

No período de outubro a dezembro de 2014 estiveram internados na UTI neonatal 18 RNs. Na tabela 1 é verificada a condição clínica da mãe, constatando-se que $16,7 \%$ destas já tiveram óbito fetal anterior, totalizando uma média de $0,28( \pm 0,73)$ óbitos fetais. A idade materna foi em média $28,1( \pm 6,3)$ anos. Das mães com neonatos internados na UTI neonatal, 11,1\% apresentavam HAS e 16,7\% diabetes gestacional. Importante ressaltar que nenhuma das mães era portadora de sífilis ou HIV. Todas as gestantes realizaram pré-natal e a média de pré-natais foi de $6( \pm 1,73)$ consultas.

Tabela 1. Condições clínicas das mães.

\begin{tabular}{cc}
\hline Características das gestantes & \\
\hline Óbito fetal anterior & $3(16,7)$ \\
Número de óbitos fetais anteriores, média $( \pm \mathrm{DP})$ & $0,28(0,73)$ \\
Idade materna, média $( \pm \mathrm{DP})$ & $28,1(6,3)$ \\
HAS, sim & $2(11,1)$ \\
Diabetes Gestacional, sim & $3(16,7)$ \\
Consultas pré-natal & $6(1,73)$
\end{tabular}

Fonte: dados da pesquisa, 2015. Dados apresentados em média \pm desvio padrão (DP) ou frequência absoluta (n) e relativa (\%).

Quanto às condições de nascimento dos RNs, verificou-se idade gestacional média de $34,4( \pm 3,11)$ semanas, o peso médio de nascimento foi de $2.260,4( \pm 687,9)$ gramas. Entre os RNs estudados verificou-se $5,6 \%$ da amostra AIG (adequado para a idade gestacional) e 5,6\% GIG (grande para idade gestacional), não sendo encontrado RN classificado como PIG (pequeno para a idade gestacional). Quanto ao índice de vitalidade, verificou-se APGAR de $6,89( \pm 2,42)$ pontos no primeiro minuto e $7,9( \pm 2,2)$ no quinto minuto (tabela 2$)$. Entre os 3.623 recém-nascidos internados em UTIN, 654 (18,1\%) evoluíram para óbito no período neonatal, durante a internação na unidade. Quase um quarto dos recém-nascidos (152/654-23,2\%) evoluiu para óbito nas primeiras 24 horas e $68,5 \%$ (448/654) desses óbitos ocorreram na primeira semana de vida do recém-nascido. 
Tabela 2. Condições de nascimento dos neonatos.

\begin{tabular}{|c|c|}
\hline Características dos RNs & \\
\hline $\mathrm{AIG}, \operatorname{sim}, \mathrm{n}(\%)$ & $1(5,6)$ \\
\hline GIG, sim, n(\%) & $1(5,6)$ \\
\hline Peso ao nascer (gramas), média ( $\pm D P)$ & $2.260,4(687,9)$ \\
\hline Idade gestacional (semanas), média ( $\pm \mathrm{DP}$ ) & $34,4(3,11)$ \\
\hline Apgar $1^{\circ}$ minuto, média ( $\left.\pm \mathrm{DP}\right)$ & $6,89(2,42)$ \\
\hline Apgar 5ํ minuto, média ( $\pm \mathrm{DP})$ & $7,9(2,2)$ \\
\hline
\end{tabular}

Fonte: dados da pesquisa, 2015. Dados apresentados em média \pm desvio padrão (DP) ou frequência absoluta (n) e relativa (\%).

Os motivos de internação dos neonatos na UTI foram em sua maioria relacionados a insuficiência respiratória $(72,2 \%)$, sepse $(22 \%)$ e condições cardiovasculares $(5,6 \%)$. Os recém-nascidos internados que ficaram em ventilação mecânica representam 33,3\% da amostra e utilizaram ventilação mecânica em média $1,9( \pm 2,9)$ dias. O uso de capuz de $\mathrm{O} 2$ aconteceu em $61,1 \%$ dos neonatos, enquanto a sedação foi utilizada em $33,3 \%$ dos neonatos. A fototerapia foi utilizada em $11,1 \%$ dos neonatos e a duração da internação na UTI foi em média 7,9 $( \pm 4,9)$ dias (tabela 3).

Buscou-se verificar possíveis associações entre idade gestacional e condições clínicas dos neonatos, no entanto, não se observou associação entre idade gestacional e motivo de internação, uso de ventilação mecânica, uso de capuz de O2 ou uso de sedação. Porém há associação entre uso de fototerapia e idade gestacional, verificando-se idade gestacional estatisticamente menor entre neonatos que utilizaram fototerapia $(29,5 \pm 6,3 ; p=0,017)$. Esses dados são apresentados na tabela 4.

Tabela 3. Condições Clínicas na UTI.

\section{Condições Clínicas}

Motivos de Internação, n(\%)

Insuficiência Respiratória

$13(72,2)$

Sepse

$4(22,2)$ 


\section{Condições Clínicas}

\begin{tabular}{|c|c|}
\hline Cardiovascular & $1(5,6)$ \\
\hline VM, sim, n(\%) & $6(33,3)$ \\
\hline Capuz de $\mathrm{O}_{2}, \operatorname{sim}, \mathrm{n}(\%)$ & $11(61,1)$ \\
\hline Uso de sedação, sim, n(\%) & $6(33,3)$ \\
\hline Fototerapia, sim, n(\%) & $2(11,1)$ \\
\hline Dias em VM, média ( $\pm \mathrm{DP})$ & $1,9(2,9)$ \\
\hline Duração da internação na UTI, média ( $\pm D P)$ & $7,9(4,9)$ \\
\hline
\end{tabular}

Fonte: dados da pesquisa, 2015. Dados apresentados em média \pm desvio padrão (DP) ou frequência absoluta (n) e relativa (\%).

Tabela 4. Associação entre idade gestacional e condições do neonato na UTI.

\begin{tabular}{|c|c|c|}
\hline Condições Clínicas & Idade Gestacional & Valor de $p$ \\
\hline \multicolumn{3}{|l|}{ Motivos de Internação } \\
\hline Insuficiência Respiratória & $34,5( \pm 1,9)$ & \multirow{3}{*}{0,829} \\
\hline Sepse & $33,8( \pm 6,5)$ & \\
\hline Cardiovascular & $36(-)$ & \\
\hline \multicolumn{3}{|l|}{ Ventilação Mecânica } \\
\hline Sim & $34( \pm 4,5)$ & \multirow[t]{2}{*}{0,694} \\
\hline Não & $34,6( \pm 2,2)$ & \\
\hline \multicolumn{3}{|l|}{ Capuz de $\mathrm{O}_{2}$} \\
\hline Sim & $34,4( \pm 1,9)$ & \multirow{2}{*}{0,993} \\
\hline Não & $34,4( \pm 5,6)$ & \\
\hline \multicolumn{3}{|l|}{ Uso de sedação, sim } \\
\hline Sim & $34,3( \pm 3,2)$ & \multirow{2}{*}{0,875} \\
\hline Não & $34,5( \pm 2,3)$ & \\
\hline \multicolumn{3}{|l|}{ Fototerapia } \\
\hline Sim & $29,5( \pm 6,3)$ & \multirow{2}{*}{$0,017^{*}$} \\
\hline Não & $35( \pm 2,3)$ & \\
\hline
\end{tabular}

Fonte: dados da pesquisa, 2015. Dados apresentados em média \pm desvio padrão (DP) ou frequência absoluta (n) e relativa (\%). ${ }^{*}$ indica diferença estatisticamente significativa $(p<0,05)$. 
Tabela 5. Desfecho Clínico, n(\%).

\begin{tabular}{cc}
\hline Desfechos & \\
\hline Alta, $\mathrm{n}(\%)$ & $16(88,9)$ \\
Óbito, $\mathrm{n}(\%)$ & $1(5,6)$ \\
Transferência, $\mathrm{n}(\%)$ & $1(5,6)$
\end{tabular}

Fonte: dados da pesquisa, 2015. Dados apresentados em frequência absoluta (n) e relativa (\%).

\section{DISCUSSÃO}

Este estudo demonstrou que as principais causas de internação na UTIN foram insuficiência respiratória, sepse e cardiovascular. As mães realizaram em torno de 6 consultas pré-natal. $O$ estudo de Lima et al. ${ }^{4}$ demonstra que as principais causas de internação em UTI neonatal são prematuridade, afecções respiratórias e baixo peso e que a maioria das mães realizaram entre 1 e 5 consultas de pré-natal.

Neste estudo observou-se um percentual considerável de mães com história de aborto fetal anterior. Montenegro Jr. et al. ${ }^{7}$ definem aborto como óbito fetal que ocorre antes da $20^{\mathrm{a}}$ semana de gestação. A incidência de aborto dentre diabéticas é controversa. De forma geral, tem sido considerada como semelhante à da população geral, que está em torno de $10 \%$. Contudo, estudos prospectivos têm demonstrado frequências mais altas dessa complicação, entre 15\% e 30\% em gestantes com diabetes mellitus do tipo 1 sendo relacionados principalmente à hiperglicemia no período pré-concepção. Comparando com esse resultado observou-se neste estudo que das 18 gestantes (16,7\%) apresentaram diabetes gestacional. Então, a realização de consultas no pré-natal é de grande importância, melhorando assim a assistência à saúde durante a gestação, evitando complicações tardias ${ }^{5,6}$. Montenegro Jr. et al. ${ }^{7}$ observaram que algumas medidas podem contribuir com a redução de complicações, como a frequência dos retornos, na maioria das pacientes de 1 a 2 semanas durante todo o seguimento; internações nos casos mal controlados, buscando uma monitorização glicêmica razoável; a disponibilidade para aquisição gratuita da insulina na rede pública de saúde; a persistência na dietética, a cada retorno e durante a 


\section{Atenção à Saúde}

internação; a utilização e ajuste da insulinoterapia nos casos não controlados com a $\operatorname{dieta}^{7}$.

Nesta pesquisa as gestantes realizaram em média 6 consultas de pré-natal. Um bom acompanhamento com pré-natal e esclarecimento sobre a importância de um bom parto, em que se conhece as condições do bebê, ganho de peso fetal, batimentos fetais, são realizados exames complementares, pode diminuir as chances de internação em UTI e consequentemente o risco de óbito para a mãe e o recémnascido ${ }^{5,6}$.

Barbosa et al. (2014) verificou a ocorrência de sepse precoce em 8,5\% dos neonatos internados em UTI e esta foi associada a alta mortalidade ${ }^{8}$. Já Silva et al (2014) mostram como principais fatores associados a mortalidade em UTI-N Apgar menor que 7 no quinto minuto, uso de tubo endotraqueal e oligodramnia ${ }^{9}$. $O$ estudo de Barbosa et al. ${ }^{8}$ classificou o Apgar menor que 7 no quinto minuto de vida como um fator de risco para sepse precoce. Segundo Eberle et al. ${ }^{10} \mathrm{o}$ baixo valor do índice de Apgar é muito empregado como sinônimo de asfixia neonatal. Porém o Apgar deve refletir as condições de vitalidade do recém-nascido e, juntamente com outras variáveis, faz parte do indicador near miss neonatal, que identifica fatores preditores de morte ${ }^{11,12}$. É importante ressaltar que no presente estudo a média de Apgar no primeiro minuto foi menor que 7 , e no quinto minuto ficou em torno de 7 .

No estudo de Silva et al. ${ }^{9}$ verificou-se o uso de VM com máscara em $72,9 \%$ dos sobreviventes e $27,1 \%$ dos RNs não sobreviventes. O uso de tubo endotraqueal aconteceu em $58,3 \%$ dos sobreviventes e $41,7 \%$ dos não sobreviventes. Essa pesquisa sugere que $33,3 \%$ do RNs fizeram uso de ventilação mecânica durante a internação na UTI. Durante os três anos de estudo foram internados na UTIN 198 recém-nascidos. Destes, 13 foram desconsiderados na análise estatística por não possuírem informações relevantes para o estudo ou por terem sido transferidos para outro serviço, com perda de seguimento. Desse modo, foram incluídos no estudo 185 neonatos, sendo que 19 (10,3\%) foram a óbito antes do $28^{\circ}$ dia de vida. A necessidade de ventilação mecânica ocorreu em $43,7 \%$ da população ${ }^{13}$. Nesta pesquisa pode-se observar que $33,3 \%$ do RNs fizeram uso de ventilação mecânica durante a internação na UTI. Na pesquisa de Oliveira et al. ${ }^{14}$ nenhum dos casos da extubação ocorreu por falta de sedação. Os sinais clínicos que levaram à determinação do evento de extubação não planeada (ENP) foram: choro audível, em $26,8 \%$ dos casos; 
Artigo Original

\section{Atenção à Saúde}

exteriorização do tubo, em $26,8 \%$ dos casos; cianose, em $23,2 \%$ dos casos; piora do padrão respiratório, em $12,2 \%$ dos casos; conteúdo gástrico no tubo endotraqueal (TET), em 7,3\% dos casos; e bradicardia, em 3,7\% dos casos.

No presente estudo $11 \%$ dos RNs fizeram uso de fototerapia, sendo que houve associação entre a menor idade gestacional e necessidade de fototerapia $(p=0,017)$. Punaro, Mezzacappa e Facchini ${ }^{15}$ demonstraram que durante a primeira semana de vida, $18,9 \%$ dos RNs foram reinternados para fototerapia. Os valores médios da bilirrubina total máxima atingida e seu momento de ocorrência foram, respectivamente, $20,2 \pm 2,2 \mathrm{mg} / \mathrm{dL}$ e $125,1 \pm 49,5$ horas. A ocorrência de $B T \geq 25 \mathrm{mg} / \mathrm{dL}$ é evento raro, ocorrendo em $0,15 \%$ dos nascimentos. A alta mais precoce não foi identificada como fator associado à hiperbilirrubinemia, embora a alta entre 48-72 horas de vida possa aumentar em duas vezes a chance de reinternação, comparativamente à alta mais tardia. $\mathrm{O}$ baixo aporte recebido pode ser apontado como principal causa de icterícia neonatal ${ }^{16}$.

Esta pesquisa apresenta algumas limitações, quais sejam tempo e amostra reduzidos. Porém, ainda assim, é possível perceber as condições de nascimento e evolução clínica dos RNs que necessitam de cuidados intensivos no período neonatal.

\section{CONSIDERAÇÕES FINAIS}

Neste estudo verificou-se que a principal causa de internação na UTIN foi insuficiência respiratória, sepse e cardiovascular. As mães realizaram em torno de 6 consultas pré-natal e a idade gestacional foi estatisticamente menor entre neonatos que utilizaram fototerapia, revelando o quão importante é o acompanhamento ambulatorial, realizando as consultas do pré-natal desde o início da gestação, evitando transtornos para o bebê e para os pais.

Assim sendo, este estudo evidencia a importância dos cuidados pré-natais, apesar de não pesquisá-los diretamente, pois percebe-se a influência dos cuidados recebidos neste período sobre os recém-nascidos que necessitam de internação em UTIN. Além dos cuidados pré-natais, destaca-se a necessidade de uma equipe treinada para o cuidado do neonato, bem como que esta tenha a sensibilidade de atender também os pais deste, que vivem um momento delicado, em alguns casos inesperado. Quando se trata do cuidado ao neonato internado em UTIN, o cuidado 
Artigo Original

Atenção à Saúde

prestado se estende para além do neonato, englobando também seus familiares. Desta forma, sugere-se que mais pesquisas sejam realizadas no âmbito da UTIN, que abordem tanto o neonato quanto seus familiares. Ressalta-se também a importância de conhecer a história gestacional da mãe, no sentido de entender parte do que ocorre com o RN internado em UTIN.

\section{REFERÊNCIAS}

1. Bustamante TF, Gonçalves TA, Ferreira G, Moraes AG. Estudo sobre a mortalidade em UTI neonatal de um hospital escola no sul de Minas. Rev ciênc saúde. 2014;4(2):1-11.

2. Nascimento LFC, Almeida MCS, Gomes CMS. Causas evitáveis e mortalidade neonatal nas microrregiões do estado de São Paulo. Rev bras ginecol obstet. 2014;36(7):303-9.

3. Barbosa AP. Terapia intensiva neonatal e pediátrica no Brasil: o ideal, o real e o possível. J pediatr. 2004;80(6):437-8.

4. Lima SS, Silva SM, Avila PES, Nicolau MV, Neves PFM. Aspectos clínicos de recém-nascidos admitidos em Unidade de Terapia Intensiva de hospital de referência da Região Norte do Brasil. ABCS health sci. 2015;40(2):7.

5. Costa ALRR, Araujo Junior E, Lima JWO, Costa FS. Fatores de risco materno associados à necessidade de unidade de terapia intensiva neonatal. Rev bras ginecol obstet. 2014;36(1):29-34.

6. Basso CG, Neves ET, Silveira A. Associação entre realização de pré-natal e morbidade neonatal. Texto contexto - enferm. 2012;21(2):269-76.

7. Montenegro Jr RM, Paccola GMFG, Faria CM, Sales APM, Montenegro APDR, Jorge SM, et al. Evolução materno-fetal de gestantes diabéticas seguidas no HCFMRP-USP no período de 1992-1999. Arq bras endocrinol metab. 2001;45(5):467-74.

8. Barbosa NG, Reis H, Resende DS, Álvares JR, Abadallah VOS, Gontijo Filho PP. Sepse neonatal precoce em unidade de terapia intensiva neonatal de um hospital universitário terciário. Pediatr mod. 2014;50(4).

9. Silva CF, Leite AJM, Almeida NMGS, Leon ACMP, Olofin I. Fatores associados ao óbito neonatal de recém-nascidos de alto risco: estudo multicêntrico em Unidades Neonatais de Alto Risco no Nordeste brasileiro. Cad saúde pública. 2014;30(2):35568.

10. Eberle AS, Ganem EM, Módolo NSP, Amorim RB, Nakamura G, Marques CDO, et al. Interação entre a analgesia de parto e o seu resultado: avaliação pelo peso e índice de Apgar do recém-nascido. Rev bras anestesiol [online]. 2006;56(4):343-51. 
11. Carmo Leal M, Silva AA, Dias MA, Gama SG, Rattner D, Moreira ME, et al. Birth in Brazil: national survey into labour and birth. Reprod health. 2012;9(15):8.

12. Avenant T. Neonatal near miss: a measure of the quality of obstetric care. Best practice \& research Clinical obstetrics \& gynaecology. 2009;23(3):369-74.

13. Lanfranchi LMMM, Viola GR, Nascimento LFC. Uso da regressão de Cox para estimar fatores associados a óbito neonatal em UTI privada. Rev paul pediatr. $2011 ; 29(2): 224-30$.

14. Oliveira PCR, Cabral LA, Schettino RC, Ribeiro SNS. Incidência e principais causas de extubação não planejada em unidade de terapia intensiva neonatal. Rev bras ter intensiva. 2012;24(3):230-5.

15. Punaro E, Mezzacappa MA, Facchini FP. Acompanhamento sistematizado da hiperbilirrubinemia em recém-nascidos com 35 a 37 semanas de idade gestacional. J pediatr. 2011;87(4):301-6.

16. Enk I, Abegg MP, Alves RJV, Stringhani F, Campos JF, Menezes HS, et al. Icterícia como causa de internação neonatal: a experiência em um serviço terciário de Porto Alegre, RS. Rev AMRIGS. 2009;53(4):361-7. 